\title{
Use of histomorphometry in the assessment of fatal vertebral artery dissection
}

\author{
C P Johnson, W Lawler, J Burns
}

\begin{abstract}
Aim-To assess morphometrically the structural changes, which occur with ageing, along the length of the vertebral artery.

Methods-A series of 36 vessels were removed at necropsy from subjects aged between 9 months and 86 years. Image analysis was used to measure the medial width, the circumference, the intimal: medial area ratio and the adventitial: medial area ratio along each artery. The artery from a case of fatal vertebral artery dissection, which occurred after a game of cricket and then chiropractic neck manipulation, was also examined in the same manner. The proteoglycan accumulation in the media was quantified using an eyepiece graticule.

Results-The vertebral arteries were, on average, larger around the origin of the vessel from the subclavian artery, and the adventitia were relatively thicker at this point, and also after piercing the dura mater. The media were much thinner within the intracranial segment and pronounced intimal thickening occurred with increasing age. The dissected artery showed undoubtable pre-existent structural abnormalities, in the form of massive proteoglycan accumulation, which predisposes an artery to dissection.

Conclusions-These data should help pathologists faced with the task of assessing the underlying structural integrity of the vessel wall in cases of vertebral artery injury.
\end{abstract}

( Clin Pathol 1993;46:1000-1003)

Vertebral artery dissection is rare and can occur without a known history of neck trauma ${ }^{12}$ and in the absence of any apparent underlying vascular disease. In most instances, however, there is a documented injury to the neck, often of a trivial nature. ${ }^{3}$ For example, chiropractic manipulation, which in some countries is a popular treatment for neck disorders, has led to neurological impairment as a result of damage to the vertebral artery. In such cases the vertebral artery lesion is suspected clinically and the diagnosis confirmed either by angiography, ${ }^{4}$ computed tomography scan, ${ }^{5}$ or magnetic resonance imaging scan. ${ }^{6}$ The condition may resolve spontaneously or it may result in variable residual disability. Fatal cases documented with thorough post mortem examination and histological examination are exceptional.
The underlying state of the artery before manipulation is of great medicolegal importance with regards to negligence, and the paucity of published data on the morphology of the vertebral artery has obviously hampered such investigation. We have recently documented, by a simple point counting method, the patterns of proteoglycan accumulation that occur with age along the length of this transitional artery. ${ }^{7}$

\section{Methods}

Eighteen pairs of vertebral arteries were removed from normal subjects, aged 9 months to 86 years, at post mortem examination. ${ }^{8}$ Ten annuli, $0.5 \mathrm{~cm}$ wide, were removed from each artery at the sites depicted in fig 1 . After fixation in formaldehyde, processing, and wax embedding a section from each annulus was stained with Miller's elastin van Gieson (EVG), giving a total of 360 sections, which were assessed morphometrically using a Seescan image analyser calibrated with a stage graticule (Graticules Ltd.). Each section was then assessed in the following way. The circumference was measured by tracing the external elastic lamina or, if this was absent,

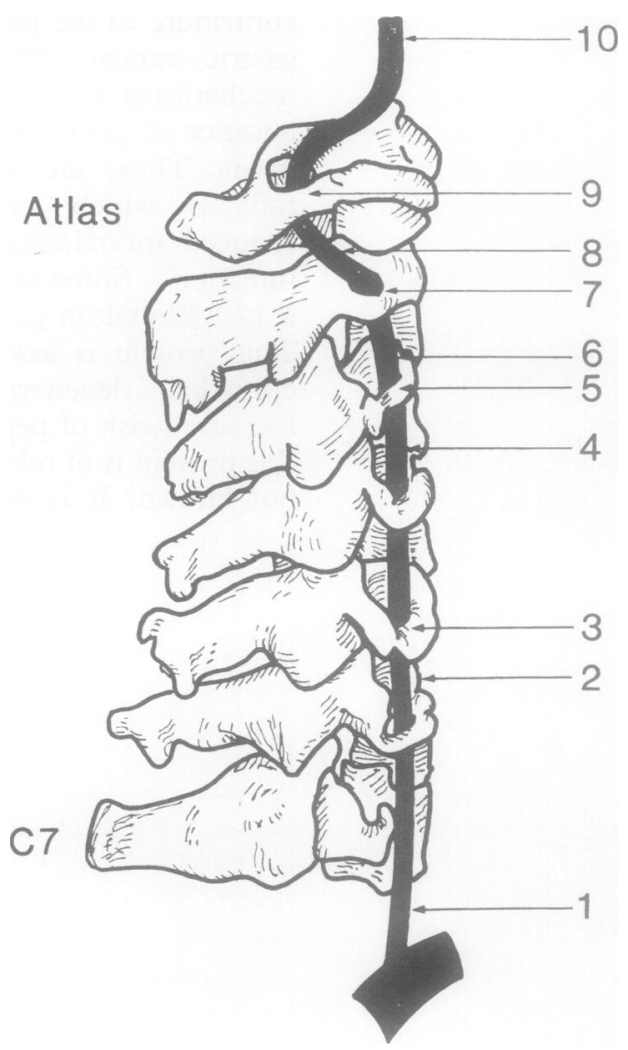

Figure 1 Diagram showing levels in vertebral artery from which sections were taken. Level 10 is just after vessel has penetrated the dura mater. 
the external boundary of the tunica media. The width of the media was measured at five random points around the section and an average value taken. To access the relative proportions of the vessel wall occupied by intima, media, and adventitia, the respective areas were traced so that the intimal:medial area ratio and the adventitial:medial area ratio could be calculated.

\section{Results}

Because no significant differences were shown between measurements taken from left or right-sided arteries, the data are not presented separately.

Figure 2 shows the average circumference plotted against level of artery, each point representing the mean of 36 values. The artery was larger adjacent to its origin from the subclavian artery. There was no significant difference in circumference with age in subjects who had reached skeletal maturity (data not shown).

Figure 3 shows the average medial widths similarly plotted against level. The tunica media was, on average, wider adjacent to its origin and thinner in the intracranial portion. No age trend was identified (data not shown).

Figure 4 shows the average intimal:medial ratio for each artery plotted against the age of the subject. There was an obvious age related increase in the intimal area, with the intimal:medial area ratio approaching 0.5 by about the age of 60 years. In only one artery did the ratio approach 1.0 . No particular level of the artery seemed to be more prone to intimal thickening (data not shown).

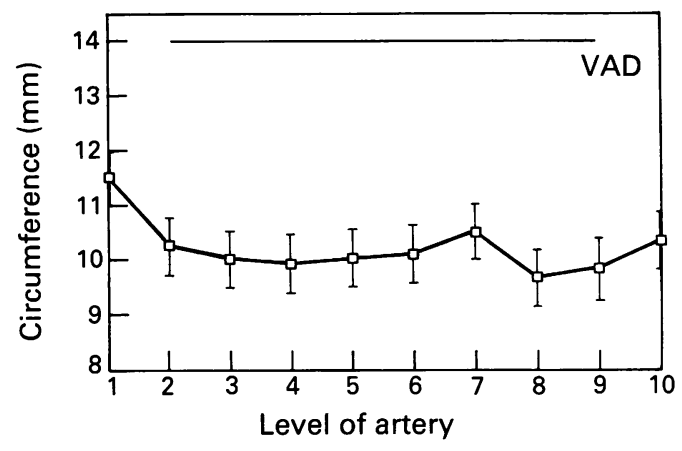

Figure 2 Graph showing circumference plotted against level of artery. VAD represents measured value in dissected vertebral artery.

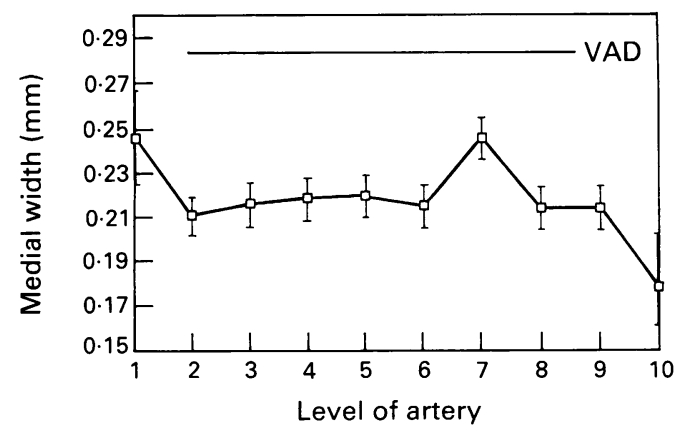

Figure 3 Graph showing medial width plotted against level of artery. VAD represents measured value in dissected vertebral artery.
Figure 5 shows the average adventitial: medial area ratio against level of the artery. The adventitia is relatively thicker adjacent to the subclavian artery and obviously so, after just penetrating the dura mater, within the intracranial portion of the vessel. This is probably a consequence of dural collagen fibres reinforcing the adventitia at this point. ${ }^{9}$ No age related changes were shown.

\section{Case report}

A 44 year old man who was previously fit and healthy, first developed problems while bowling during a cricket match. He had not played cricket since his early twenties. He sustained acute onset of pain around his left shoulder and neck during, or shortly after, this particular game. As the pains persisted, he went to see a chiropractor, two or three days later, who undertook a rotational manipulation of his neck with some apparent benefit. About five days later he suddenly developed vertigo which lasted for about four days before resolving spontaneously. The vertigo recurred the following day, became more severe, and was associated with double vision, tinnitus, left orbital headache, vomiting and weakness of the left arm. He returned to the chiropractor who referred him immediately to the local hospital. He was admitted to hospital but died before investigations could be completed. This was 15 days after his neck manipulation and about 18 days since his game of cricket.

At post mortem examination, the abnormalities were confined to the brain and the

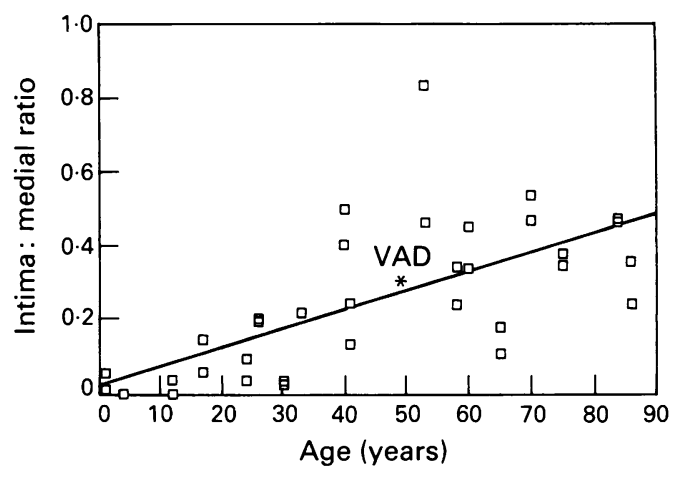

Figure 4 Graph showing intimal:medial area ratio plotted against age of subject. VAD represents measured value in dissected vertebral artery.

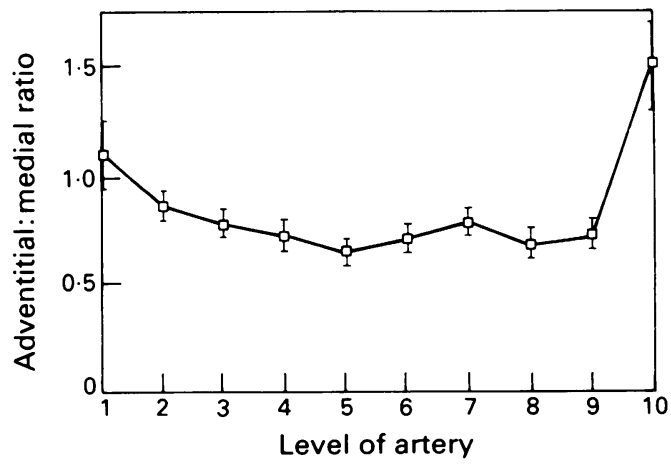

Figure 5 Graph showing adventitial:medial area ratio against level of artery. 
vertebral arteries. The cerebellum showed obvious recent infarction and was swollen and oedematous. No subarachnoid haemorrhage was present. The right vertebral artery was extremely hypoplastic. The left vertebral artery was grossly abnormal in the extracranial (cervical) segment (fig 6). Widespread arterial dissection was obvious, being most pronounced at the ends of the vessel. The intramural haemorrhage was brown and friable at the lower end of the vessel (fig 7), but of a more recent dark red appearance at the upper end (fig 8).

Sections from multiple levels were stained with haemotoxylin and eosin, alcian blue $(\mathrm{pH}$ 2.5), and Miller's elastin van Gieson (EVG). Histological examination confirmed a widespread intramural dissection (fig 9) and demonstrated the presence of granulation tissue at the edges of the intramural thrombus (fig 10). A large amount of alcian blue positive material with the characteristic "bubbly" appearance of proteoglycans was seen within the tunica media along the whole length of the vessel. This was quantified using an eyepiece graticule and point counting technique, as described before. ${ }^{7}$ The values obtained are the highest we have encountered in any vertebral artery, being two and a half to three times greater than those found in subjects aged 30 to 50 .

The sections stained with EVG were assessed morphometrically as above, avoiding the dissected areas. The average value for the circumference was $14.03 \mathrm{~mm}$ and for the

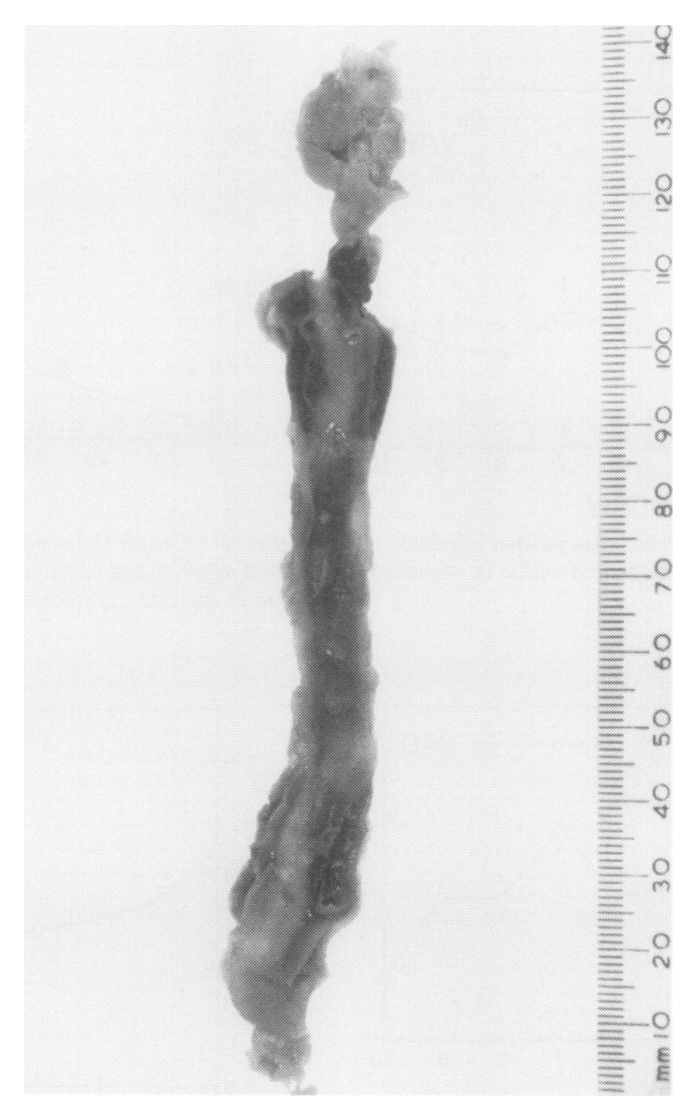

Figure 6 Opened extracranial portion of vertebral artery showing widespread arterial dissection which is most prominent at upper and lower ends.

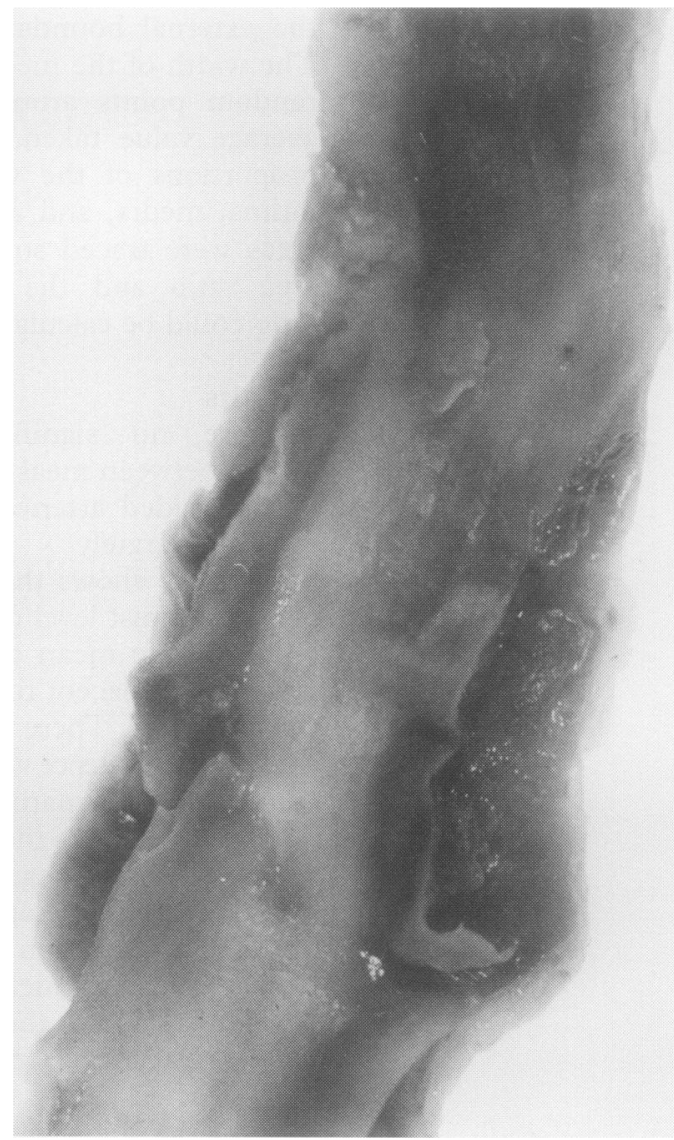

Figure 7 Lower end of artery with friable light brown haematoma within wall.

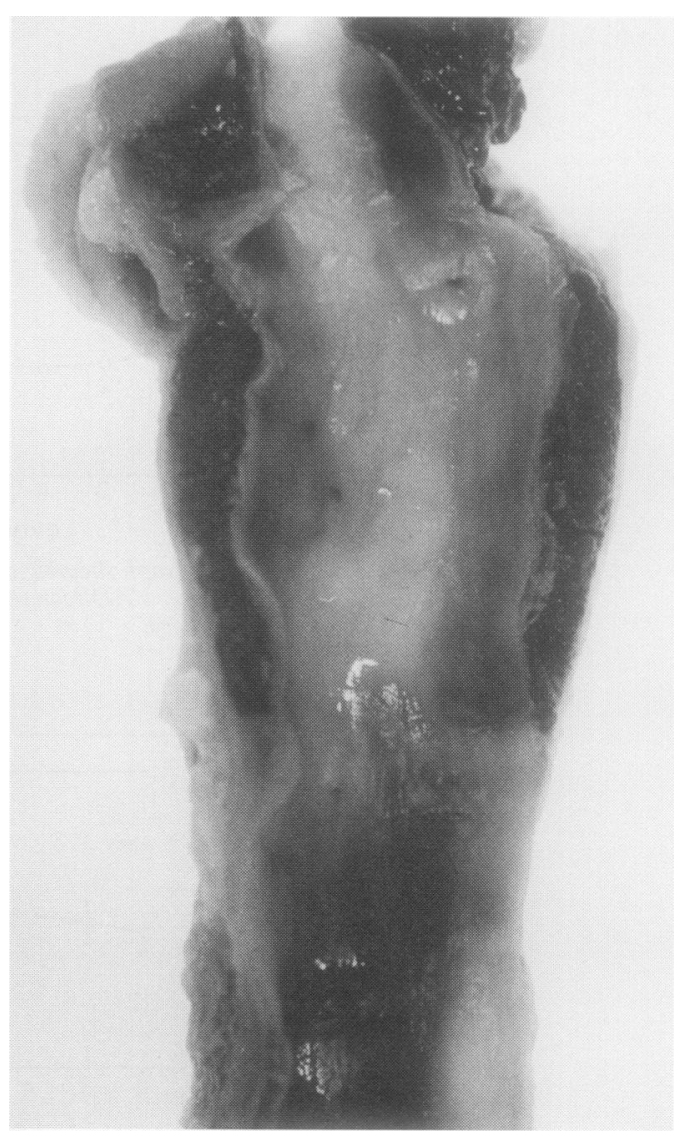

Figure 8 Upper end of artery with haematoma of darkred appearance. 


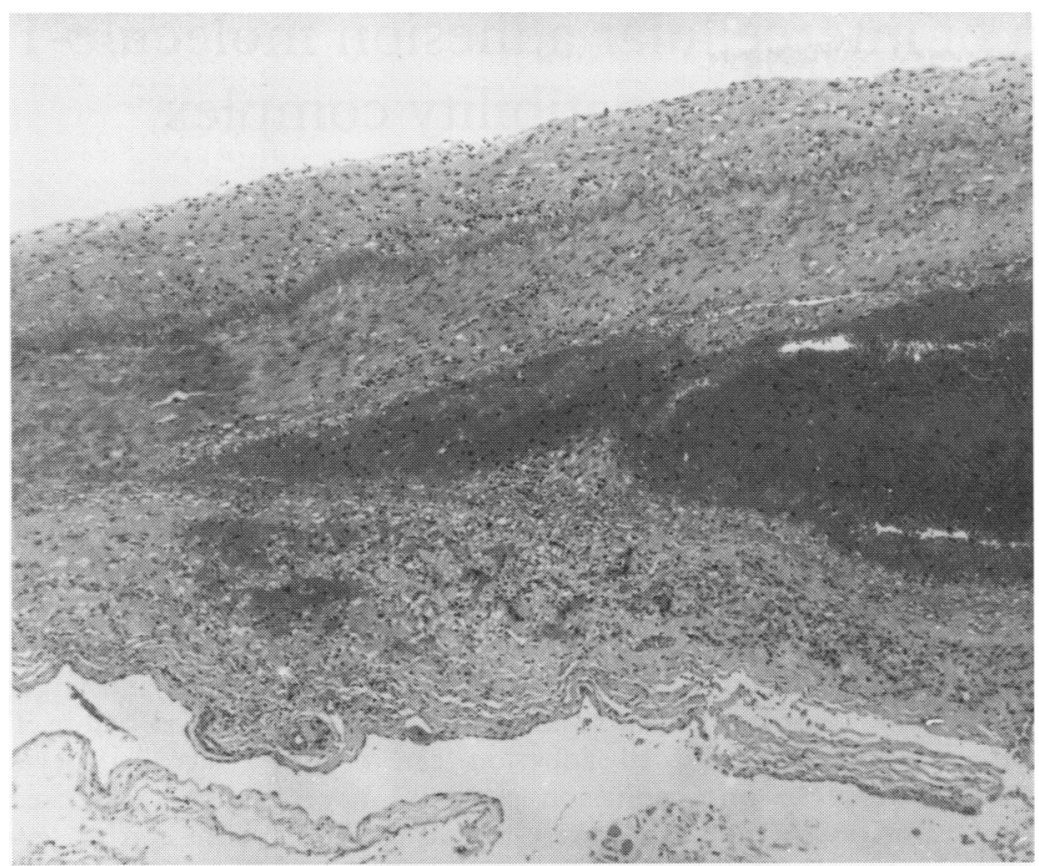

Figure 9 Section of artery confirming intramural dissection through outer part of muscular media. Adventitia is towards bottom of figure (haematoxylin and eosin).

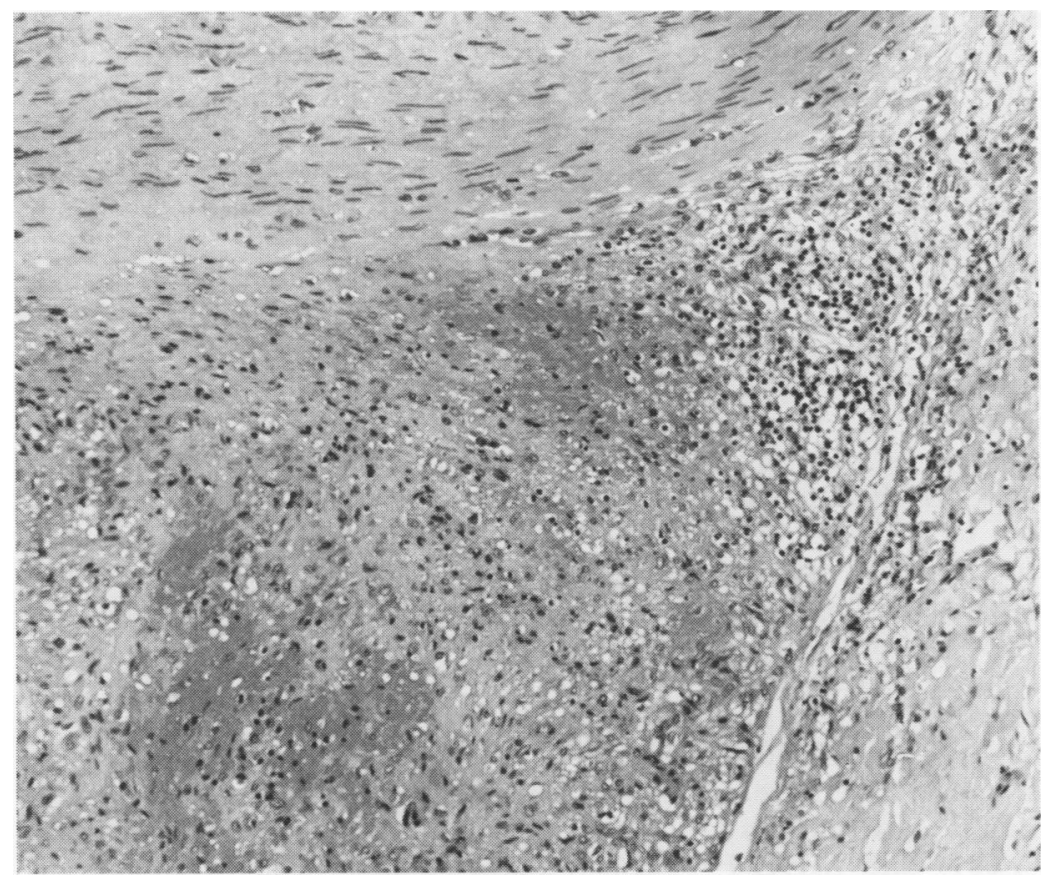

Figure 10 Well established granulation tissue around and within haematoma. Media is towards top of field and adventitia to right (haematoxylin and eosin).

media $0.288 \mathrm{~mm}$. The average intimal:medial area ratio was 0.289 and the average adventitial:medial area ratio was $0 \cdot 689$. These results have been indicated on figs 2-4 for ease of comparison but not on figure 5 as the adventitial:medial ratio result would directly overlie the existing plotted line. These show that the vessel was large and thick walled, the circumference and medial width being at the upper end of the normal range for age and site (figs 2 and 3). The proportion of the wall occupied by the intima and adventitia was normal (figs 4 and 5).

\section{Discussion}

This case raises several questions of medicolegal importance. The first is whether the arterial damage was caused by the sporting injury, the neck manipulation, or by both, or whether it occurred spontaneously. The time interval between these events and death was between 18 and 15 days and, despite the naked eye appearance of varying ages of intramural thrombus, this was not confirmed by histological examination, which shows well established granulation tissue along the entire dissection. A more recent haemorrhage into the existing dissection is, however, a possibility. The question of whether the artery was normal before the precipitating event is more easily addressed. Using morphometry, we have shown that the artery was large and had a thick muscular layer when compared with a series of 36 arteries taken from subjects of varying age. On these criteria one might expect the vessel to have been mechanically strong, although there are no experimental data to support this. What is clear, however, is that this artery showed massive accumulation of proteoglycans within the tunica media and this represents an example of the condition variably named as cystic mucoid degeneration or medial cystic necrosis, which is accepted as a predisposing cause of arterial dissection. This condition has been reported in association with vertebral artery damage, but the diagnostic criteria used in these cases are not known. ${ }^{1011}$

This man certainly had vertebral artery disease before his neck manipulation and the role of this procedure in the sequence of events remains speculative. Some of the other reported cases of vertebral artery dissection might have had morphological abnormalities which would not be readily recognised with routine methods of investigation and in which quantitative techniques might help to explain -for example, why only a small percentage of people who undergo neck manipulation die.

We thank Mr A Williams, Mrs J Holtom, and Miss S Anderson for their help in the preparation of this article.

1 Chen J, Smith $R$, Keller A, Kucharcyzk W. Spontaneous dissection of the vertebral artery: MR Findings. $f$ Comput Assist Tomogr 1989;13:326-9.

2 Youl BD, Coutellier A, Dubois B, Leger JM, Bousser MG. Three cases of spontaneous extracranial vertebral artery dissection. Stroke 1990;21:618-25.

$3 \mathrm{Manz}$ HJ, Luessenhop AJ. Dissecting aneurysm of intracranial vertebral artery: case report and review of the literature. $\mathcal{F}$ Neurol 1983;230:25-35.

4 Greselle JF, Zenteno M, Kien P, Castel JP, Caille JM. Spontaneous dissection of the vertebro-basilar system. $f$ Neuroradiol 1987;14:115-23.

5 Power T, Hanlon D, Brillman J. Dissections of the cervicocerebral arteries. Ann Emerg Med 1991;20:1032-5.

6 Gelbert F, Assouline E, Hodes JE, et al. MRI in spontaneous dissection of vertebral and carotid arteries. Neuroneous dissection of vertebral

7 Johnson CP, Burns J. The medico-legal significance of ohnson CP, Burns J. The medico-legal significance of proteoglycans in the tunica media of the vert

8 Bromilow A, Burns J. A technique for removal of the vertebral arteries. $f$ Clin Pathol 1985;38:1400-2.

9 Wilkinson IMS. The vertebral artery. Extracranial and intracranial structure. Arch Neurol 1972;27:392-6.

10 Ide Y, Fukushima T, Yamamoto M, Tomonaga M. Vertebral dissecting aneurysm associated with medial mucoid degeneration. Neurol Med Chir (Tokyo) 1986; 26:888-94.

11 Sherman MR, Smialek JE, Zane WE. Pathogenesis of vertebral artery occlusion following cervical spine manipulation. Arch Pathol Lab Med 1987;111:851-3. 